\title{
Study on the Integration of Network Information Resources in Digital Library
}

\author{
Yanbo Wang \\ Library, Xi'an University , Xi'an, Shaanxi, PR. China 710065
}

\begin{abstract}
Keywords: Digital library; Network Information Resources; Integration; Approach
Abstract. This study analyzed the definition of the digital library, as well as the characteristics and conception of the network information resources, based on which the contents of integration of the network information resources in digital library and three approaches of integration which are the establishment of the resource directory, the integration of the search engine and the processing of the metadata, have been discussed as well.
\end{abstract}

\section{Introduction}

With the increasing number of the introduction of network database in university libraries and the effect of search engine on reading habits of reader, there is a huge change in the document resources construction and digital service in libraries, which also makes it a focus of attention for university libraries that how to fully utilize the network resources in library. At present, many libraries have already integrated the network resources with the traditional library resources in different degree to enhance the efficient use of electronic document resources and make it easier for readers to quickly search, use and access to digital resources and traditional documents. As a consequence, how to integrate the network information resources in digital library remains a big issue that should be taken into consideration in the tide of informatization today.

\section{The definition of the digital library}

Digital library, namely, electronic library, is a virtual form of library, which is also referred to as online library. There are two mainstreams for the explanation of the digital library definition, which are Britain and America. Back to the early 1990s, American digital library alliance has already defined digital library for the first time. They believed that digital library is an organization for providing documents or human resources which also aims at collecting, accessing, transferring and utilizing the digital data. In contrary, scholars in Britain insisted that digital library is the extraction, storage and management for information and resources through digital technique, without the consideration of the publication form. While in China, we considered that digital library is a virtual form of electronic library based on the computer and internet technology by taking the way of internet online communication, which provides information of books and documents for readers, integrates the network information resources, creates a breakthrough of restrictions of time and space for resources and make it more convenient for the users to use.

\section{The basic conception and the characteristics of the network information resources}

The connotation and the classification of the network information resources. With the development of various information technologies, the network information resources have become a great wealth buried in the information era. Even that, the current definition of the network information resources is still very abstract, and there is not a clear and specific boundary. This study believes that the network information resources can be considered the overall available information resources in internet system for users to use.

In terms of the classification of the network information resources, there are different ways to classify according to different references. For example, taking timeliness for classification, they can 
be classified into full-text periodicals, electronic newspapers, dynamic form and bibliographic records, etc.; By taking various forms of media for classification, they can be classified into text, audio, image, video and three-dimensional type, etc.; If communication mode is taken for classification, they can be classified into informal publication and formal publication; And according to the different levels of the network information resources, they can be classified into deictic information, unit of information, documents, system of the network information resources, and so on. All in all, the network information can make people's life more convenient, but there is harmful information that will possibly mislead people's philosophy and value at the same time.

The necessity and principles of integration of the network information resources. First of all, in terms of the user experience, both the accuracy and the practicability of integration of the network information resources need to be enhanced, which aims to improve the convenience for users to use. With the gradually increasing the network information, as far as the digital library be concerned, there is a great need for them to keep in step with the situation, change the traditional way of thinking on the integration of information, take into consideration the characteristics of the network information resources such as diversification and irregularity, and reorganization and integration of the network information resources need to be carried out so as to maintain the essential and leave the unessential. Second, with the development of information technology, a trend of saving and managing the network information resources is the automation of the network information organization, which in other words, is the development for a shift from traditional manual reorganization to the automation techniques. An unescapable result and requirement of the automation of network resources integration are the need to integrate the network information, in a way of enhancing the speed and efficiency. Last but not least, integration of network resources can greatly improve the accuracy of the network information through reorganization of classification in diverse levels, forms and types, which meets the need of users in a better way due to an extremely large amount of information that the network information possesses. Based on the objective need for quickly search and higher accuracy, digital library must thoroughly carry out the integration of the network information resources.

The integration of the network information resources can't be conducted blindly and without the consideration of actual situation. It must in line with some certain rules. First, the correlation of documents and person need to be fully taken into consideration for enhancing the vitality of library documents during the integration. Second, the ideological level and quality of user can be improved through thorough and profound classification and selecting. And for the next step, the professionalization of resources classification in library need to be ensured and the level of service in library need to be constantly improved. Last but not least, more effort on the resource searching in terms of accuracy need to put in so as to make it convenient for users to search and use.

Contents of the integration of the network information resources in digital library. In China, the contents of integration of the network information resources in digital library so far can be specifically classified into the following kinds, which are zeroth literature, initial information, secondary information and third information.

The zeroth information is also referred to as grey literature. This kind is of high specialty with a low rate of reading by readers and low pellucidity. Even that, it plays a very important role in the network information resources, which is the base of the network information. Given that the zeroth information includes various characteristics such as high quality, updated information, high specialty and large amount of information, in the overall process of integration, the relative database of zeroth information need to be established for providing the readers long-distance searching and reading.

The initial information mainly refers to the large amount of initial information present on the internet. In view of the different source channels of initial information which presents in different regions, it contains various types and points to different characteristics, leading to very complicated contents of initial information on the internet and irregularity of quality. Therefore, during the integration of initial information, the practical value of initial information need to be analyzed and then classified based on the high and low order of value. The invalid and useless information need to 
be deleted. As for the high-practicability information, they can be integrated and processed by using hypertext.

Considering that the internet has no ability to distinguish and transfer the information itself, there is no way to classify and select those complicated information on the internet. As a consequence, it is necessary to integrate the main tool during the integration of initial information on the network at present, which is the search engine. The rate of utilization and efficiency of these initial information can be enhanced by establishing the network information retrieval tools. Hence, regarding to those initial information that have never been processed, they can be defined as secondary information through some specific means or ways such as manual processing or semi-automatic processing and being refined and accumulated into a sort of documents with function of tool.

On the network, characteristics and functions are divers in different search engine. During the process of information retrieval through using the search engine by users, different methods are required. Therefore, in consideration of being more convenient for users to utilize the search engine during information retrieval, libraries need to integrate those search engine, which can make it more quick and accurate to retrieve and use the information for users. The result of reintegration for the secondary information turns them into third information. The priority for all in the integration of the network information resources in digital library today, is the third information based on the database of search engine which are developed from those well-processed secondary information.

The approaches for the integration of the network information resources. Integration of resource directory. The so call network information resource directory, in its essence, is a sort of main guideline similar with those physical libraries, but it can only be applied in the virtual form of digital library. The general steps for conducting the integration of the network information resources directory are as follows. First of all, manual or semi-automatic approach is used for regulating and classify those selected information. Next, the selected information is classified in some sort of standard. Last but no least, different information is abstracted regarding to different standard and set in the frame that is suitable for the various features of information, thus the directory is formed. As regarding to the large book directory of internet, it is noteworthy that all the contents of information will not be able to be saved by the network information resource directory during the process of integration of the information base due to the extremely large amount of the information base of the network information. Therefore, what will be saved by the network information resource directory is the site URL or domain name of information source and a part of data related to the information such as the abstract of the content. The integration of information resource directory will develop a small-scale information database, and for the reason that the information of database saved is professionally selected, the information resource directory is of very high availability, which can improve the quality of information and enhance the specialty of organizing information. Also for the reason that the information resource directory is of rigorous systematicness and practicability, the utilization of the network information resource directory is more convenient.

The integration of search engine. At present, regarding to the utilization of search engine, it is more and more wide-spread with the popularization of the internet. Therefore, the integration of the network information resources based on the search engine is of great necessary, which needs to starting with the main contents and takes the approaches of organizing classification and indexing in order to deeply and profoundly extract the information. The analysis and selection for the contents of information from the point of view of users are also required, by which the retrieved information can be highly in line with the need of users. The search engine of network can be classified into three kinds. The first kind is directory search engine, and the second kind is keywords search engine. The third kind is meta-search engine.

The search engine is the best and first choice of tool for the network information retrieval, as well as an important method in the integration of the network information. The search engine has good characteristics for information integration, which means it can achieve a very efficient rate of retrieval. The search engine can regularly and automatically collect and absorb the relative information on the sites and can automatically label, cite and catalog for the information. Then it also can set the information into the database at the same time, analyze and extract the information and 
then establish the index database, which thereby make it possible for the search engine to provide the retrieved information and restrict the contents of retrieval.

The process of metadata. Regarding to the processing of the information in the digital library, it can be described from two aspects, which are the contents and the formation of the information and it can originally and profoundly demonstrate them as well. The description by formation mainly refers to the description of the external characteristics for the outer image of the information. And as for the description by contents, it mainly refers that the language retrieval is as the standard by information resources, and this kind of description can deeply and profoundly demonstrate the internal characteristics of the contents of information. The digital library need to establish a system in the level of the network information, which can be used to label and describe the external and internal characteristics of the information, and as a consequence, the information can be realized and can appear as a complete visage of great efficiency.

In conclusion, it is of great necessity that the deep development of the digital library is carried out based on the profound integration of the network resources, for which it should persistently start with reality, and the profundity and extent of the integration will be guided by the scientific technology and scientific theory. It is also necessary to collect, process and regulate the information on the network, take the only standard based on the experience of users for the inspection of the integration, enhance its own ability of creation and sense of service, increase the training of library workers, conduct the integration of resources with a more serious attitude and more ground-breaking vision, which can make it more professional to integrate the resources, and make it more practical and convenient. By which is mentioned above, the digital library can develop in a fast speed, and the utilization of the digital library will be more and more wide-spread, which can improve the literacy and the level of life quality for users in China, enhance the convenience and efficiency as well so as to ensure that China is at the forefront of the construction of internet.

\section{References}

[1] Chen, Y.Z, Analysis on relative problems of integration of network information resources in art universities, Journal of Nanjing Art Institute (Art and design), (6), pp. 164-167, 2013.

[2] Xu, R.Z, Application of cloud computing technology in the integration service of network information resources, Practical Electonics, (11), pp. 163-163,164, 2014.

[3] Zong, Y, Integration of network information resources in university libraries based on the teaching and research, Scientific Advisory, (7), pp. 70-71, 2013.

[4] Ge, X.X, Lv, Y, Yan, Y, Analysis of integration of academic network information resources taking website of foreign agricultural science community as an example, Library Theory and Practice, (1), pp. 9-62, 2015.

[5] Qiao HQ, Strategy analysis of integration of network information resources under the background of the construction of digital library, A Vast View on Publishing, (8), 2015.

[6] Chen, F, You, L.G, Investigation and analysis of current situation of integration of information resources of municipal archives website in Shandong Province, Shandong Archives, (5), 2013.

[7] Hong, Q, Analysis of contents and mode utilized in integration of website archives information resources, Decision and Information (Midmonth), (8), 2015.

[8] Chu, Q.Q, Analysis of current situation of network information organizing investigation in China, Journal of Library Sicence, (3), 2008.

[9] Guo, Q, Analysis of new-type integration of resources in digital library under the condition of internet, Academic Education, 2012. 
[10] Huang, Y, Analysis of integration of network information resources in digital library, Informatization Construction, 2015. 